\title{
AN EXTENSION OF POSITIVITY FOR INTEGRALS OF BESSEL FUNCTIONS AND BUHMANN'S RADIAL BASIS FUNCTIONS
}

\author{
YONG-KUM CHO, SEOK-YOUNG CHUNG, AND HERA YUN
}

(Communicated by Mourad Ismail)

Abstract. As to the Bessel integrals of type

$$
\int_{0}^{x}\left(x^{\mu}-t^{\mu}\right)^{\lambda} t^{\alpha} J_{\beta}(t) d t \quad(x>0),
$$

we improve known positivity results by making use of new positivity criteria for ${ }_{1} F_{2}$ and ${ }_{2} F_{3}$ generalized hypergeometric functions. As an application, we extend Buhmann's class of compactly supported radial basis functions.

\section{INTRODUCTION}

We consider the problem of determining positivity of the integrals

$$
\int_{0}^{x}\left(x^{\mu}-t^{\mu}\right)^{\lambda} t^{\alpha} J_{\beta}(t) d t \quad(x>0)
$$

for appropriate values of parameters $\mu, \lambda, \alpha, \beta$, where $J_{\beta}$ stands for the Bessel function of order $\beta$. For the sake of convergence and practical applications, it is common to assume $\mu>0, \lambda \geq 0, \beta>-1, \alpha+\beta+1>0$.

Owing to various applications, the problem has been studied by many authors over a long period of time and we refer to Askey [1] and Gasper [7] for historical backgrounds. Of our primary concern is the result of Misiewicz and Richards which states in a simplified version as follows.

Theorem A (Misiewicz and Richards [14]). Let $\mathcal{A}$ be the set of parameters $(\beta, \alpha)$ defined by

$$
\mathcal{A}=\left\{\beta>-\frac{1}{2},-\beta-1<\alpha \leq \min \left(\beta, \frac{3}{2}\right)\right\} .
$$

For $0<\mu \leq 1$ and $\lambda \geq 1$, if $(\beta, \alpha) \in \mathcal{A}$, then

$$
\int_{0}^{x}\left(x^{\mu}-t^{\mu}\right)^{\lambda} t^{\alpha} J_{\beta}(t) d t>0 \quad(x>0) .
$$

An additional range of parameters $\alpha, \beta$ is also available. In fact, if $j_{\beta, 2}$ denotes the second positive zero of $J_{\beta}$ and $\alpha_{*}(\beta)$ the solution of

$$
\int_{0}^{j_{\beta, 2}} t^{\alpha_{*}(\beta)} J_{\beta}(t) d t=0
$$

Received by the editors December 18, 2017, and, in revised form, January 8, 2018.

2010 Mathematics Subject Classification. Primary 33C20, 41A30, 42B10.

Key words and phrases. Bessel function, Fourier transform, generalized hypergeometric function, Newton diagram, radial basis function, positivity.

(C) 2018 by the authors under Creative Commons Attribution-Noncommercial 3.0 License (CC BY NC 3.0) 
for each $\beta$, then Misiewicz and Richards pointed out the above positivity holds true for $-1<\beta<\frac{1}{2},-\beta-1<\alpha<\alpha_{*}(\beta)$. As it is described in detail by Askey [2], however, the explicit nature of $\alpha_{*}(\beta)$ is still unknown and we shall exclude this range throughout.

In the special case $\alpha=\frac{1}{2}, \beta=-\frac{1}{2}$, the integrals of (11) reduce to the Fourier cosine transforms for which Kuttner proved its positivity:

Theorem B (Kuttner [12]). For $0<\mu \leq 1$ and $\lambda \geq 1$,

$$
\int_{0}^{x}\left(x^{\mu}-t^{\mu}\right)^{\lambda} \cos t d t>0 \quad(x>0) .
$$

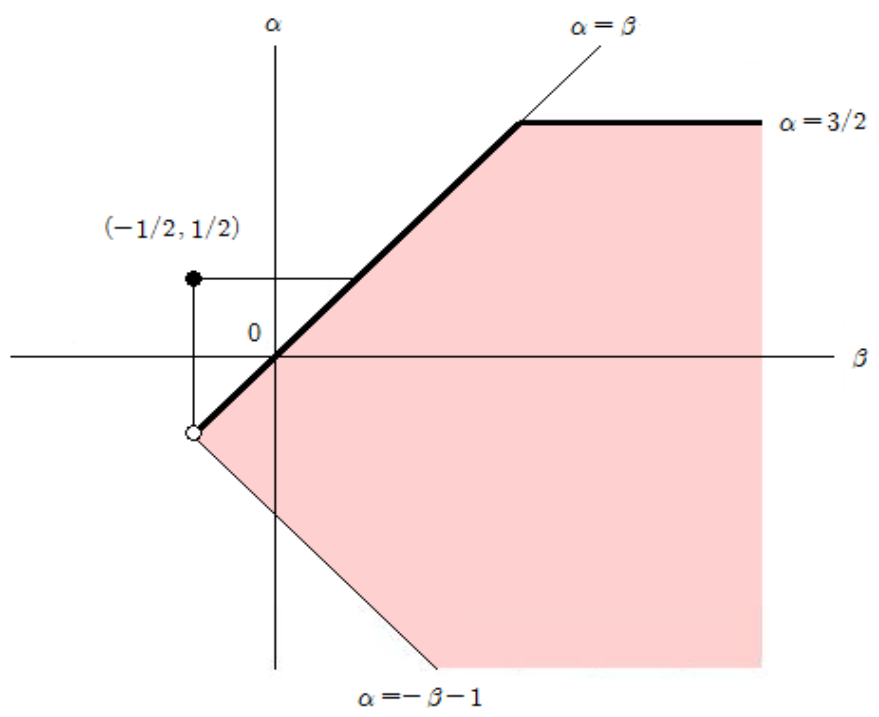

FiguRE 1. The positivity regions of Misiewicz-Richards (pink) and Kuttner (black dot)

The combined positivity region is depicted in Figure 1 .

The main purpose of the present paper is to improve Theorem A and Theorem B by extending positivity regions for $0<\mu \leq 1, \lambda \geq 1$ as well as by providing a positivity region for unrestricted $\mu>0, \lambda \geq 0$.

As an application of our results, we shall improve in several directions the range of positive definiteness for Buhmann's class of compactly supported radial basis functions [4] which are of considerable interest in the theory of approximations and interpolations.

\section{Positivity in the unrestricted CASE}

For $\alpha, \beta$ satisfying $\beta>-1, \alpha+\beta+1>0$, if we put

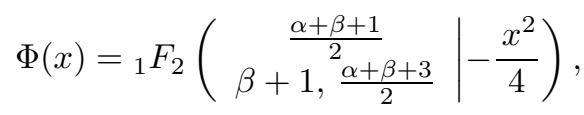


then it is simple to evaluate by integrating termwise or by parts

$$
\begin{aligned}
& \int_{0}^{x} t^{\alpha} J_{\beta}(t) d t=\frac{x^{\alpha+\beta+1}}{2^{\beta} \Gamma(\beta+1)(\alpha+\beta+1)} \Phi(x) \\
& \int_{0}^{x}\left(x^{\mu}-t^{\mu}\right)^{\lambda} t^{\alpha} J_{\beta}(t) d t \\
& \quad=\frac{\mu \lambda x^{\mu \lambda+\alpha+\beta+1}}{2^{\beta} \Gamma(\beta+1)(\alpha+\beta+1)} \int_{0}^{1} \Phi(x t)\left(1-t^{\mu}\right)^{\lambda-1} t^{\mu+\alpha+\beta} d t
\end{aligned}
$$

for $\mu>0, \lambda>0$ and $x>0$. Therefore positivity of (11) would follow once kernel $\Phi$ were shown to be positive in the case $\lambda=0$ or nonnegative in the case $\lambda>0$.

To investigate the sign of ${ }_{1} F_{2}$ generalized hypergeometric function $\Phi$, we shall make use of the following general criterion recently established by Cho and Yun [5], which will be applied subsequently in other occasions as well.

As it is standard, the Newton diagram associated to a finite set of planar points $\left\{\left(\alpha_{i}, \beta_{i}\right): i=1, \cdots, N\right\}$ refers to the closed convex hull containing

$$
\bigcup_{i=1}^{N}\left\{(x, y) \in \mathbb{R}^{2}: x \geq \alpha_{i}, y \geq \beta_{i}\right\} .
$$

Lemma 2.1. (Cho and Yun, [5]) For $a>0, b>0, c>0$, put

$$
\phi(x)={ }_{1} F_{2}\left(a ; b, c ;-\frac{x^{2}}{4}\right) \quad(x>0) .
$$

(i) If $\phi \geq 0$, then necessarily $b>a, c>a, b+c \geq 3 a+\frac{1}{2}$.

(i) Let $P_{a}$ denote the Newton diagram associated to

$$
\Lambda=\left\{\left(a+\frac{1}{2}, 2 a\right),\left(2 a, a+\frac{1}{2}\right)\right\} .
$$

$$
\text { If }(b, c) \in P_{a} \text {, then } \phi \geq 0 \text { and strict positivity holds unless }(b, c) \in \Lambda \text {. }
$$

For the sake of presenting this paper in a self-contained way, we shall give a simplified proof in the appendix. Keeping in mind that the line segment joining two point of $\Lambda$ is given by $c=3 a+1 / 2-b$ in the $(b, c)$-plane, it is straightforward to obtain the range for the positivity or nonnegativity of $\Phi$ by implementing Lemma 2.1 .

Theorem 2.1. Let $\mathcal{R}$ be the set of parameters $(\beta, \alpha)$ defined by

$$
\mathcal{R}=\{\beta>-1,-\beta-1<\alpha \leq 0\} \cup\left\{\beta>0,0<\alpha \leq \min \left(\beta, \frac{1}{2}\right)\right\} .
$$

For $\mu>0, \lambda \geq 0$ and $(\beta, \alpha) \in \mathcal{R}$, we have

$$
\int_{0}^{x}\left(x^{\mu}-t^{\mu}\right)^{\lambda} t^{\alpha} J_{\beta}(t) d t>0 \quad(x>0)
$$

unless $\lambda=0, \alpha=\beta=1 / 2$. In the exceptional case, it reduces to

$$
\int_{0}^{x} J_{\frac{1}{2}}(t) \sqrt{t} d t=\frac{2 \sqrt{2}}{\sqrt{\pi}} \sin ^{2}\left(\frac{x}{2}\right) \geq 0 .
$$




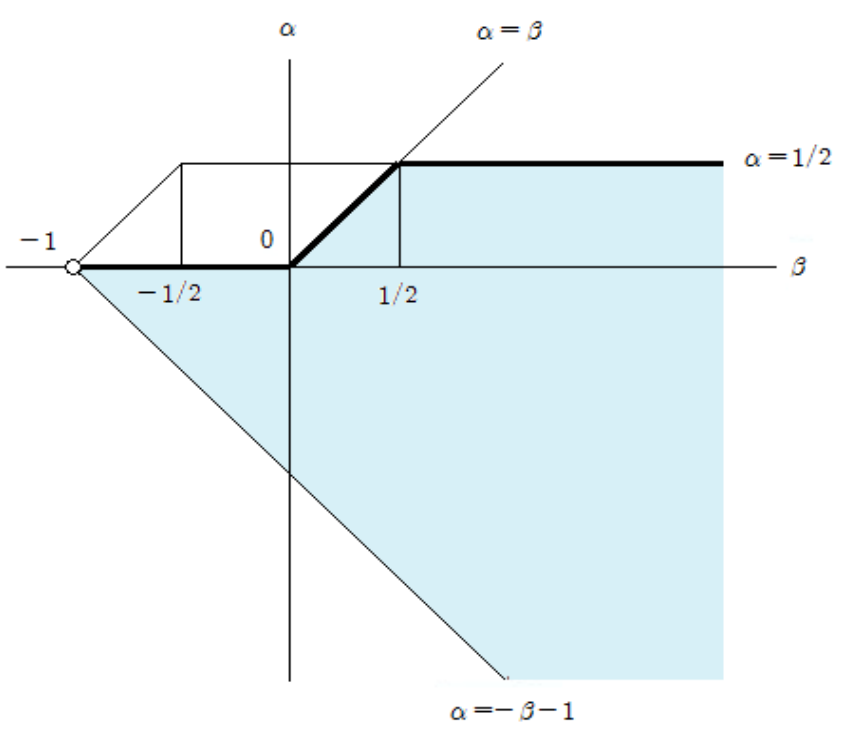

FIGURE 2. The positivity region in the unrestricted case $\mu>$ $0, \lambda \geq 0$.

Remark 2.1. Geometrically, $\mathcal{R}$ represents an infinite polygonal region depicted as in Figure 2. In the case $\lambda=0$, it follows from an inspection on Lemma 2.1 that the necessity region for nonnegativity is given by $\{\beta>-1,-\beta-1<\alpha \leq 1 / 2, \alpha<\beta+1\}$ so that our result does not cover the parallelogram defined by

$$
\{0<\alpha \leq 1 / 2, \beta<\alpha<\beta+1\} .
$$

Proof. For the positivity of $\Phi$, we write $A=(\alpha+\beta+1) / 2$ and apply Lemma 2.1 with $a=A, b=A+1, c=\beta+1$. For $0<A<1 / 2$, $\Phi$ is positive when $\beta+1 \geq 2 A$, that is, $-\beta-1<\alpha<-\beta, \alpha \leq 0$. For $A \geq 1 / 2, \Phi$ is positive when $\beta+1 \geq \max (2 A-1 / 2, A+1 / 2)$, that is, $-\beta \leq \alpha \leq \min (\beta, 1 / 2)$. Combining, we obtain the stated region of positivity.

In the special case $\mu=2, \lambda>0$, positivity region $\mathcal{R}$ of Theorem 2.1 can be improved considerably. As a matter of fact, if we observe

$$
\int_{0}^{x}\left(x^{2}-t^{2}\right)^{\lambda} t^{\alpha} J_{\beta}(t) d t=\frac{B\left(\lambda+1, \frac{\alpha+\beta+1}{2}\right) x^{2 \lambda+\alpha+\beta+1}}{2^{\beta+1} \Gamma(\beta+1)} \Psi(x),
$$

where $B$ stands for the Euler's beta function and

$$
\Psi(x)={ }_{1} F_{2}\left(\begin{array}{c}
\frac{\alpha+\beta+1}{2} \\
\beta+1, \lambda+1+\frac{\alpha+\beta+1}{2}
\end{array} \mid-\frac{x^{2}}{4}\right),
$$

then it is routine to deduce from Lemma 2.1 the following result.

Theorem 2.2. Let $\mathcal{S}$ be the set of parameters $(\beta, \alpha)$ defined by

$$
\mathcal{S}=\{\beta>-1,-\beta-1<\alpha \leq 0\} \cup\left\{\beta>0, \alpha \leq \min \left(\beta, \lambda+\frac{1}{2}\right)\right\} .
$$


If $\lambda>0$ and $(\beta, \alpha) \in \mathcal{S}$, then

$$
\int_{0}^{x}\left(x^{2}-t^{2}\right)^{\lambda} t^{\alpha} J_{\beta}(t) d t>0 \quad(x>0)
$$

unless $\alpha=\beta=\lambda+1 / 2$ for which the integral reduces to

$$
\int_{0}^{x}\left(x^{2}-t^{2}\right)^{\lambda} t^{\lambda+\frac{1}{2}} J_{\lambda+\frac{1}{2}}(t) d t=\frac{\sqrt{\pi} \Gamma(\lambda+1)\left(2 x^{2}\right)^{\lambda+\frac{1}{2}}}{2} J_{\lambda+\frac{1}{2}}^{2}\left(\frac{x}{2}\right) \geq 0 .
$$

Remark 2.2. In [7, Gasper also obtained a positivity region in this case. Our result, however, is an improvement in that the triangle with vertices $(0,0)$, $(\lambda+1 / 2,0),(\lambda+1 / 2, \lambda+1 / 2)$ is missing in Gasper's positivity region.

\section{Positivity OF ${ }_{2} F_{3}$ Hypergeometric functions}

While Newton diagrams give positivity regions of ${ }_{1} F_{2}$ hypergeometric functions, it appears that there are no such criteria available for ${ }_{2} F_{3}$ hypergeometric functions. Our purpose here is to develop some criteria of positivity, which will be exploited later on.

As usual, we shall use Pochhammer's notation to denote

$$
(\alpha)_{k}=\alpha(\alpha+1) \cdots(\alpha+k-1), \quad(\alpha)_{0}=1
$$

for any real number $\alpha$ and positive integer $k$. We refer to Bailey [3], and Luke [13] for definitions and basic properties of generalized hypergeometric functions.

A basic feature on positivity is the following.

Lemma 3.1. For positive real numbers $a, b, c, d, e$, suppose that

$$
{ }_{2} F_{3}\left(\begin{array}{c|c}
a, b \\
c, d, e
\end{array} \mid-\frac{x^{2}}{4}\right)>0 \quad(x>0) .
$$

Then for any $\delta \geq 0, \gamma \geq 0, \epsilon \geq 0$, we also have

$$
{ }_{2} F_{3}\left(\begin{array}{c|c}
a, b \\
c+\delta, d+\gamma, e+\epsilon
\end{array} \mid-\frac{x^{2}}{4}\right)>0 \quad(x>0) .
$$

Proof. Assuming $\delta>0$, we have

$$
\begin{aligned}
& { }_{2} F_{3}\left(\begin{array}{c}
a, b \\
c+\delta, d, e
\end{array} \mid-\frac{x^{2}}{4}\right) \\
& =\frac{2}{B(c, \delta)} \int_{0}^{1}{ }_{2} F_{3}\left(\begin{array}{c}
a, b \\
c, d, e
\end{array} \mid-\frac{x^{2} t^{2}}{4}\right)\left(1-t^{2}\right)^{\delta-1} t^{2 c-1} d t>0
\end{aligned}
$$

and the other cases follow in the same manner or by symmetry.

We next deal with ${ }_{2} F_{3}$ hypergeometric functions of the form

$$
\begin{aligned}
\Omega(x) & ={ }_{2} F_{3}\left(\begin{array}{c}
a, a+\frac{1}{2} \\
c+1, a+b, a+b+\frac{1}{2} \mid
\end{array} \mid-\frac{x^{2}}{4}\right) \\
& =\frac{1}{B(2 a, 2 b)} \int_{0}^{1}{ }_{0} F_{1}\left(c+1 ;-\frac{x^{2} t^{2}}{4}\right)(1-t)^{2 b-1} t^{2 a-1} d t
\end{aligned}
$$

with parameters satisfying $a>0, b>0, c>-1$.

We apply Gasper's sums of squares formula ([7], (3.1)) to write

$$
\Omega(x)=\Gamma^{2}(\nu+1)\left(\frac{x}{4}\right)^{-2 \nu} \sum_{n=0}^{\infty} C(n, \nu) \frac{(2 n+2 \nu)}{n+2 \nu} \frac{(2 \nu+1)_{n}}{n !} J_{\nu+n}^{2}\left(\frac{x}{2}\right)
$$


in which $C(n, \nu)$ denotes the terminating series defined by

$$
C(n, \nu)={ }_{5} F_{4}\left(\begin{array}{c}
-n, n+2 \nu, \nu+1, a, a+\frac{1}{2} \\
\nu+\frac{1}{2}, c+1, a+b, a+b+\frac{1}{2}
\end{array} \mid 1\right)
$$

and $\nu$ is an arbitrary real number such that $2 \nu$ is not a negative integer.

Due to the interlacing property on the zeros of Bessel functions $J_{\nu}, J_{\nu+1}$ (see Watson [16]), the positivity of $\Omega$ would follow instantly from formula (9) if $C(n, \nu)>$ 0 for all nonnegative integers $n$ and $\nu>-1 / 2$.

Our investigation on the sign of $C(n, \nu)$ will be carried out along the following steps. We recall that a ${ }_{p} F_{q}$ generalized hypergeometric function is said to be Saalschützian if the sum of numerator parameters plus one is equal to the sum of denominator parameters.

Step 1 . We choose $\nu>-1 / 2$ in such a unique way that each coefficient $C(n, \nu)$ becomes a Saalschützian terminating series, that is,

$$
\nu=b+\frac{c}{2}-\frac{1}{4} \quad \text { with } \quad b+\frac{c}{2}+\frac{1}{4}>0 .
$$

Step 2. In [8], Gasper discovered a summation formula which states

$$
\begin{aligned}
& { }_{p+2} F_{p+1}\left(\begin{array}{c}
-n, a_{1}, \cdots, a_{p+1} \\
b_{1}, \cdots, b_{p+1}
\end{array} \mid 1\right) \\
& =\sum_{k=0}^{n}\left(\begin{array}{l}
n \\
k
\end{array}\right) \frac{\left(b_{1}+b_{2}-a_{1}-1\right)_{k}\left(b_{1}-a_{1}\right)_{k}\left(b_{2}-a_{1}\right)_{k}}{\left(b_{1}+b_{2}-a_{1}-1\right)_{2 k}} \frac{\left(a_{2}\right)_{k} \cdots\left(a_{p+1}\right)_{k}}{\left(b_{1}\right)_{k} \cdots\left(b_{p+1}\right)_{k}} \\
& \quad \times{ }_{p+1} F_{p}\left(\begin{array}{c}
k-n, k+a_{2}, \cdots, k+a_{p+1} \\
2 k+b_{1}+b_{2}-a_{1}, k+b_{3}, \cdots, k+b_{p+1}
\end{array} \mid 1\right) .
\end{aligned}
$$

An application of this formula gives

$$
\begin{aligned}
C(n, \nu) & ={ }_{5} F_{4}\left(\begin{array}{c}
-n, n+2 b+c-\frac{1}{2}, b+\frac{c}{2}+\frac{3}{4}, a, a+\frac{1}{2} \\
b+\frac{c}{2}+\frac{1}{4}, c+1, a+b, a+b+\frac{1}{2}
\end{array} \mid 1\right) \\
& =\sum_{k=0}^{n}\left(\begin{array}{l}
n \\
k
\end{array}\right) \frac{\left(2 a+b-\frac{c}{2}-\frac{5}{4}\right)_{k}\left(a-\frac{c}{2}-\frac{3}{4}\right)_{k}\left(a-\frac{c}{2}-\frac{1}{4}\right)_{k}}{\left(2 a+b-\frac{c}{2}-\frac{5}{4}\right)_{2 k}} \\
& \times \frac{\left(n+2 b+c-\frac{1}{2}\right)_{k}(a)_{k}\left(a+\frac{1}{2}\right)_{k}}{(a+b)_{k}(c+1)_{k}\left(a+b+\frac{1}{2}\right)_{k}\left(b+\frac{c}{2}+\frac{1}{4}\right)_{k}} A_{k}(a, b, c),
\end{aligned}
$$

where $A_{k}(a, b, c)$ denotes the Saalschützian series defined as

$$
A_{k}(a, b, c)={ }_{4} F_{3}\left(\begin{array}{c}
k-n, k+n+2 b+c-\frac{1}{2}, k+a, k+a+\frac{1}{2} \\
2 k+2 a+b-\frac{c}{2}-\frac{1}{4}, k+b+\frac{c}{2}+\frac{1}{4}, k+c+1
\end{array} \mid 1\right) .
$$

Step 3. We next apply Whipple's transformation formula (Bailey [3], 7.2(1)),

$$
\begin{aligned}
{ }_{4} F_{3}\left(\begin{array}{c}
-m, x, y, z \\
u, v, w
\end{array} \mid 1\right) & =\frac{(v-z)_{m}(w-z)_{m}}{(v)_{m}(w)_{m}} \\
& \times{ }_{4} F_{3}\left(\begin{array}{c}
-m, u-x, u-y, z \\
1-v+z-m, 1-w+z-m, u
\end{array} \mid 1\right)
\end{aligned}
$$


valid if it is Saalschützian, to decompose further

$$
\begin{aligned}
& A_{k}(a, b, c)=\frac{\left(b+\frac{c}{2}+\frac{1}{4}-a\right)_{n-k}(c+1-a)_{n-k}}{\left(k+b+\frac{c}{2}+\frac{1}{4}\right)_{n-k}(k+c+1)_{n-k}} \times \\
& { }_{4} F_{3}\left(\begin{array}{c}
k-n, k-n+2 a-b-\frac{3 c}{2}+\frac{1}{4}, k+a+b-\frac{c}{2}-\frac{3}{4}, k+a \\
k-n+a-b-\frac{c}{2}+\frac{3}{4}, k-n+a-c, 2 k+2 a+b-\frac{c}{2}-\frac{1}{4}
\end{array} \mid 1\right) .
\end{aligned}
$$

Step 4. From expansion formula (12), it is evident $C(n, \nu)>0$ if

$$
2 a>-b+\frac{c}{2}+\frac{5}{4}, a \geq \frac{c}{2}+\frac{3}{4}, b+\frac{c}{2}+\frac{1}{4}>0
$$

and $A_{k}(a, b, c)>0$ for all $k$. By using an elementary inequality

$$
\frac{(-m)_{j}(-m+\alpha)_{j}}{(-m+\beta)_{j}(-m+\gamma)_{j}}>0, \quad j=0,1, \cdots, m,
$$

is valid as long as $\alpha \leq 1, \beta<1, \gamma<1$, we deduce from (13) $A_{k}(a, b, c)>0$ when

$$
2 a \leq b+\frac{3 c}{2}+\frac{3}{4}, a<b+\frac{c}{2}+\frac{1}{4}, a<c+1 .
$$

Combining (14), (15), we may summarize what we have proved as follows.

Theorem 3.1. For $a>0, b>0, c>-1$, we have

$$
\Omega(x)={ }_{2} F_{3}\left(\begin{array}{c|c}
a, a+\frac{1}{2} \\
c+1, a+b, a+b+\frac{1}{2}
\end{array} \mid-\frac{x^{2}}{4}\right)>0 \quad(x>0)
$$

if $a, b, c$ satisfy the following conditions simultaneously.

$$
\left\{\begin{array}{l}
\frac{c}{2}+\frac{3}{4} \leq a<\min \left(c+1, b+\frac{c}{2}+\frac{1}{4}\right), \\
-b+\frac{c}{2}+\frac{5}{4}<2 a \leq b+\frac{3 c}{2}+\frac{3}{4} .
\end{array}\right.
$$

(i) In the boundary case $a=b+\frac{c}{2}+\frac{1}{4}$, (16) also holds true if

$$
\frac{1}{2}<b \leq \frac{c}{2}+\frac{1}{4}, c>\frac{1}{2} \text {. }
$$

(ii) In the boundary case $c=a-1$, (16) also holds true if

$$
b \geq \max \left[1, \frac{1}{2}\left(a+\frac{3}{2}\right)\right],(a, b) \neq\left(\frac{1}{2}, 1\right) .
$$

In the case $(a, b)=\left(\frac{1}{2}, 1\right)$, we have $\Omega(x) \geq 0$ for $x>0$.

Proof. It remains to prove positivity in the two boundary cases.

(i)We apply Whipple's transformation formula of Step 3 directly to obtain

$$
\begin{aligned}
C(n, \nu)= & \frac{\left(b-\frac{1}{2}\right)_{n}(b)_{n}}{\left(2 b+\frac{c}{2}+\frac{1}{4}\right)_{n}\left(2 b+\frac{c}{2}+\frac{3}{4}\right)_{n}} \\
& \times{ }_{4} F_{3}\left(\begin{array}{c}
-n,-n-2 b+\frac{3}{2},-b+\frac{c}{2}+\frac{1}{4}, b+\frac{c}{2}+\frac{3}{4} \\
-n-b+\frac{3}{2},-n-b+1, c+1
\end{array}\right),
\end{aligned}
$$

which is easily seen to be positive under the former condition by the same reasonings as in Step 4. If $b=c / 2+1 / 4, a=c+1 / 2$, then $\Omega$ reduces to

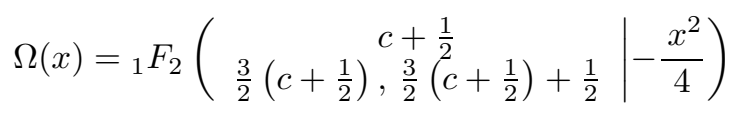


and positivity with $c>1 / 2$ follows by Lemma 2.1 (see also Fields and Ismail [6]).

(ii) In this case, it is easy to deduce again from Lemma 2.1

$$
\Omega(x)={ }_{1} F_{2}\left(\begin{array}{c|c}
a+\frac{1}{2} \\
a+b, a+b+\frac{1}{2}
\end{array} \mid-\frac{x^{2}}{4}\right)>0
$$

under the stated condition. In the case $(a, b)=\left(\frac{1}{2}, 1\right), \Omega$ reduces to

$$
\Omega(x)=\left[\frac{\sin (x / 2)}{x / 2}\right]^{2} \geq 0 .
$$

In the special case $b=1$, we obtain

Corollary 3.1. For $a>0, c>-1$, we have

$$
{ }_{2} F_{3}\left(\begin{array}{c|c}
a, a+\frac{1}{2} \\
c+1, a+1, a+\frac{3}{2}
\end{array} \mid-\frac{x^{2}}{4}\right)>0 \quad(x>0)
$$

if a,c satisfy one of the following conditions.

(i) $\left\{\begin{array}{l}\frac{c}{2}+\frac{3}{4} \leq a<\min \left(c+1, \frac{c}{2}+\frac{5}{4}\right), \\ \frac{c}{2}+\frac{1}{4}<2 a \leq \frac{3 c}{2}+\frac{7}{4} .\end{array}\right.$
(ii) $a=\frac{c}{2}+\frac{5}{4}, c \geq \frac{3}{2} . \quad$ (iii) $c=a-1,0<a \leq \frac{1}{2}$.

\section{Improved Results of Misiewicz AND Richards}

In the case $0<\mu \leq 1 \leq \lambda$, the density $t \mapsto\left(1-t^{\mu}\right)_{+}^{\lambda}$ is convex and nonincreasing on $(0, \infty)$. By using Williamson's characterization [19] on such monotone convex functions, Misiewicz and Richards [14] observed

$$
\begin{gathered}
\int_{0}^{x}\left(x^{\mu}-t^{\mu}\right)^{\lambda} t^{\alpha} J_{\beta}(t) d t=x^{\mu \lambda-1} \int_{0}^{1} K(x t) d G(t), \\
K(x)=\int_{0}^{x}(x-t) t^{\alpha} J_{\beta}(t) d t,
\end{gathered}
$$

with a unique probability measure $G$, so that the positivity of (11) under consideration would follow once kernel $K$ were shown to be nonnegative.

In view of the well-known Bessel identity (Watson [16]),

$$
J_{\beta}(x)=\frac{1}{\Gamma(\beta+1)}\left(\frac{x}{2}\right)_{0}^{\beta} F_{1}\left(\beta+1 ;-\frac{x^{2}}{4}\right), \quad \beta>-1,
$$

it is simple to modify (8) to evaluate

$$
K(x)=\frac{B(\alpha+\beta+1,2) x^{\alpha+\beta+2}}{2^{\beta} \Gamma(\beta+1)}{ }_{2} F_{3}\left(\begin{array}{c}
\frac{\alpha+\beta+1}{2}, \frac{\alpha+\beta+2}{2} \\
\beta+1, \frac{\alpha+\beta+3}{2}, \frac{\alpha+\beta+4}{2}
\end{array} \mid-\frac{x^{2}}{4}\right)
$$

and hence the problem of positivity reduces to the nonnegativity question on the ${ }_{2} F_{3}$ hypergeometric functions defined in (20).

Our improvement of Theorem A reads as follows. 
Theorem 4.1. Let $\mathcal{P}$ be the set of parameters $(\beta, \alpha)$ defined by

$$
\mathcal{P}=\left\{\beta>-1,-\beta-1<\alpha \leq \min \left[\beta+1, \frac{1}{2}\left(\beta+\frac{3}{2}\right), \frac{3}{2}\right]\right\} .
$$

For $0<\mu \leq 1 \leq \lambda$ and $(\beta, \alpha) \in \mathcal{P}$, we have

$$
\int_{0}^{x}\left(x^{\mu}-t^{\mu}\right)^{\lambda} t^{\alpha} J_{\beta}(t) d t>0 \quad(x>0) .
$$

Remark 4.1. In Figure 3, the trapezoid with vertices

$$
(-1,0),(-1 / 2,-1 / 2),(3 / 2,3 / 2),(-1 / 2,1 / 2)
$$

is newly added to the positivity region of Misiewicz and Richards which corresponds to the infinite polygon bounded by $\alpha=-\beta-1, \alpha=\beta, \alpha=\frac{3}{2}$.

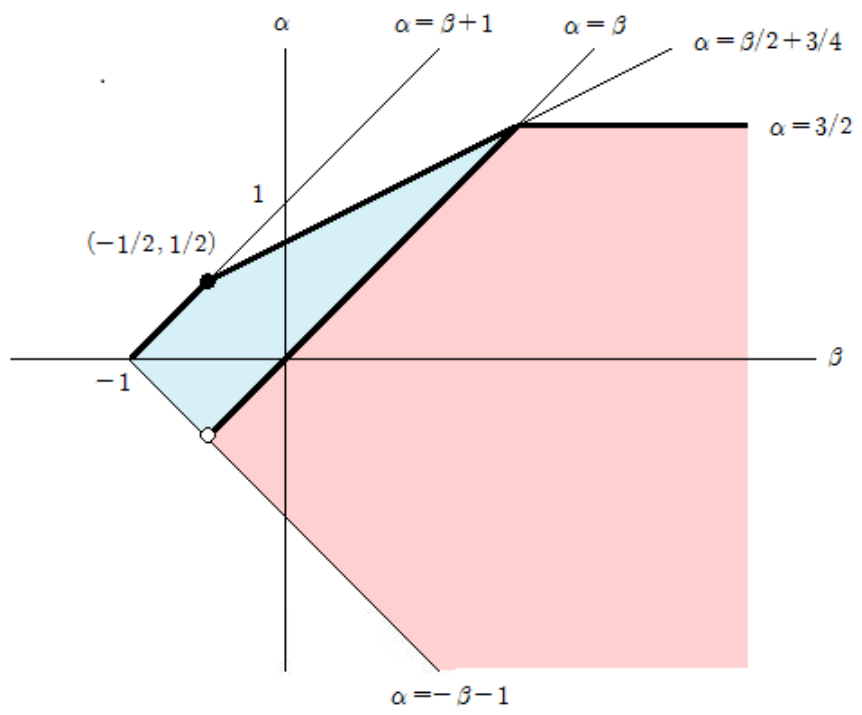

Figure 3 . The positivity region in the case $0<\mu \leq 1 \leq \lambda$ which improves the ones of Misiewicz and Richards (pink) and Kuttner (black dot).

Proof. The ${ }_{2} F_{3}$ hypergeometric functions of (20) are of type (17) with

$$
a=\frac{\alpha+\beta+1}{2}, \quad c=\beta .
$$

It is simple to find condition (i) of Corollary 3.1 gives the infinite strip

$$
\beta \geq-\frac{1}{2}, \quad \frac{1}{2} \leq \alpha<\frac{3}{2}, \quad \alpha \leq \frac{1}{2}\left(\beta+\frac{3}{2}\right)
$$

as a positivity region. Likewise, conditions (ii), (iii) correspond to the boundary lines

$$
\beta \geq \frac{3}{2}, \alpha=\frac{3}{2} \text { and } \quad-1<\beta \leq-\frac{1}{2}, \alpha=\beta+1
$$


To fill out the remaining positivity region, we observe from Lemma 3.1 that if (20) is positive with some parameters $\alpha_{0}, \beta_{0}$, then positivity continues to hold true for all parameters $\alpha_{0}-\delta, \beta_{0}+\delta, \delta \geq 0$, that is, for all $\alpha, \beta$ lying on the half-line emanating from $\left(\beta_{0}, \alpha_{0}\right)$ defined by $\alpha=-\beta+\alpha_{0}+\beta_{0}, \beta \geq \beta_{0}$. By adding all half-lines emanating from $(\beta, \alpha) \in \mathcal{P}$ constructed from (21), (22), we obtain the full stated region $\mathcal{P}$.

In the special case $\mu=1$, reduction (18) is unnecessary for

$$
\begin{aligned}
& \int_{0}^{x}(x-t)^{\lambda} t^{\alpha} J_{\beta}(t) d t=\frac{B(\alpha+\beta+1, \lambda+1) x^{\lambda+\alpha+\beta+1}}{2^{\beta} \Gamma(\beta+1)} \\
& \times{ }_{2} F_{3}\left(\begin{array}{c}
\frac{\alpha+\beta+1}{2}, \frac{\alpha+\beta+2}{2} \\
\beta+1, \frac{\alpha+\beta+\lambda+2}{2}, \frac{\alpha+\beta+\lambda+3}{2}
\end{array} \mid-\frac{x^{2}}{4}\right)
\end{aligned}
$$

in which the generalized hypergeometric functions are of type (16) with

$$
a=\frac{\alpha+\beta+1}{2}, b=\frac{\lambda+1}{2}, c=\beta .
$$

A direct application of Theorem 3.1 yields the following improved result.

Theorem 4.2. For $\lambda>0$ and $(\beta, \alpha) \in \mathcal{O}$, we have

$$
\int_{0}^{x}(x-t)^{\lambda} t^{\alpha} J_{\beta}(t) d t>0 \quad(x>0),
$$

where $\mathcal{O}$ denotes the set of parameters defined by

$$
\mathcal{O}=\left\{\begin{array}{c}
\beta>-1,-\beta+1-\lambda<\alpha \leq \min \left[\frac{1}{2}\left(\beta+\lambda+\frac{1}{2}\right), \lambda+\frac{1}{2}\right], \quad \text { if } \quad \lambda<1, \\
\beta>-1,-\beta-1<\alpha \leq \min \left[\beta+1, \frac{1}{2}\left(\beta+\lambda+\frac{1}{2}\right), \lambda+\frac{1}{2}\right], \quad \text { if } \quad \lambda \geq 1 .
\end{array}\right.
$$

As the proof proceeds in the same fashion as above, we shall omit it. We refer to Gasper [7, 8] for related results and further applications.

In view of the identity

$$
J_{-\frac{1}{2}}(t)=\sqrt{\frac{2}{\pi t}} \cos t
$$

the case $\beta=-1 / 2$ in Theorem 2.1, Theorem 4.1 corresponds to Kuttner's result.

Corollary 4.1. If $\mu>0, \lambda \geq 0,-1<\alpha \leq-\frac{1}{2}$ or $0<\mu \leq 1 \leq \lambda,-1<\alpha \leq 0$, then

$$
\int_{0}^{x}\left(x^{\mu}-t^{\mu}\right)^{\lambda} t^{\alpha} \cos t d t>0 \quad(x>0) .
$$

In the case $\alpha=0$, the problem of determining the positivity range of $\lambda=\lambda(\mu)$ is a long-standing open problem and we refer to Golubov [11] and Gneiting, Konis and Richards [10] for partial results and further references.

\section{BUHMANN'S RADIAL BASIS FUNCTIONS}

While studying scattered data approximations, Buhmann [4] introduced a 4parameter family of compactly supported radial basis functions on $\mathbb{R}^{n}$ defined as follows. 
Definition 5.1 (Buhmann's radial basis functions). For $\delta>0, \rho \geq 0, \lambda>-1$ and $\alpha>-n / 2-1$, define

$$
W(\mathbf{x})=\int_{0}^{\infty}\left(1-\frac{\|\mathbf{x}\|^{2}}{t}\right)_{+}^{\lambda} t^{\alpha}\left(1-t^{\delta}\right)_{+}^{\rho} d t \quad\left(\mathbf{x} \in \mathbb{R}^{n}\right),
$$

where $\|\mathbf{x}\|$ stands for the Euclidean norm $\|\mathbf{x}\|^{2}=\mathbf{x} \cdot \mathbf{x}$.

For this family of compactly supported radial functions, Buhmann proved the following positive definiteness (see also [20]).

Theorem $\mathbf{C}$ (Buhmann, 4]). Let $\mathcal{B}_{n}$ be the set of parameters $(\lambda, \alpha)$ defined by

$$
\begin{aligned}
\mathcal{B}_{1} & =\left\{\lambda>-\frac{1}{2},-1<\alpha \leq \min \left(\lambda-\frac{1}{2}, \frac{\lambda}{2}\right)\right\}, \\
\mathcal{B}_{2} & =\left\{\lambda>-\frac{1}{2},-1<\alpha \leq \min \left[\frac{1}{2}\left(\lambda-\frac{1}{2}\right), \lambda-\frac{1}{2}\right]\right\}, \\
\mathcal{B}_{3} & =\left\{\lambda \geq 0,-1<\alpha \leq \frac{1}{2}(\lambda-1)\right\}, \\
\mathcal{B}_{n} & =\left\{\lambda>\frac{n-5}{2},-1<\alpha \leq \frac{1}{2}\left(\lambda-\frac{n-1}{2}\right)\right\} \quad \text { if } n \geq 4 .
\end{aligned}
$$

For $0<\delta \leq \frac{1}{2}, \rho \geq 1$, if $(\lambda, \alpha) \in \mathcal{B}_{n}$, then $W$ has a strictly positive Fourier transform and hence induces positive definite matrices on $\mathbb{R}^{n}$.

As Buhmann calculated, the Fourier transform of $W$ is $\widehat{W}(\xi)=\omega(\|\xi\|), \xi \in \mathbb{R}^{n}$, where

$$
\omega(x)=\frac{(2 \pi)^{\frac{n}{2}} 2^{\lambda+1} \Gamma(\lambda+1)}{x^{n+2+2 \delta \rho+2 \alpha}} \int_{0}^{x}\left(x^{2 \delta}-t^{2 \delta}\right)^{\rho} t^{2 \alpha+1-\lambda+\frac{n}{2}} J_{\lambda+\frac{n}{2}}(t) d t
$$

for $x>0$ and Buhmann exploited Theorem A to establish the above result.

Our purpose here is to extend $\mathcal{B}_{n}$ in several directions.

We begin with extending Theorem $\mathrm{C}$ with the aid of Theorem 4.1 .

Theorem 5.1. Let $\mathcal{P}_{n}$ be the set of parameters $(\lambda, \alpha)$ defined by

$$
\mathcal{P}_{n}=\left\{\lambda>-1,-\frac{n+2}{2}<\alpha \leq \min \left[\frac{1}{4}\left(3 \lambda-\frac{n+1}{2}\right), \frac{1}{2}\left(\lambda-\frac{n-1}{2}\right)\right]\right\} .
$$

For $0<\delta \leq \frac{1}{2}, \rho \geq 1$, if $(\lambda, \alpha) \in \mathcal{P}_{n}$, then $W$ has a strictly positive Fourier transform and hence induces positive definite matrices on $\mathbb{R}^{n}$.

Remark 5.1. The proof follows trivially upon renaming parameters, that is,

$$
2 \delta \rightarrow \mu, \rho \rightarrow \lambda, 2 \alpha+1-\lambda+n / 2 \rightarrow \alpha, \lambda+n / 2 \rightarrow \beta .
$$

It is easy to observe $\mathcal{B}_{n} \subset \mathcal{P}_{n}$ in a proper way, as shown in Figure 4 in the one-dimensional case. In addition, an inspection on the two boundary lines of $\mathcal{P}_{n}$ reveals

$$
\mathcal{P}_{n}=\left\{\lambda>-1,-\frac{n+2}{2}<\alpha \leq \frac{1}{2}\left(\lambda-\frac{n-1}{2}\right)\right\} \quad \text { for } \quad n \geq 5 .
$$

In the case $\delta=1 / 2$, Theorem 4.2 gives an improvement. 


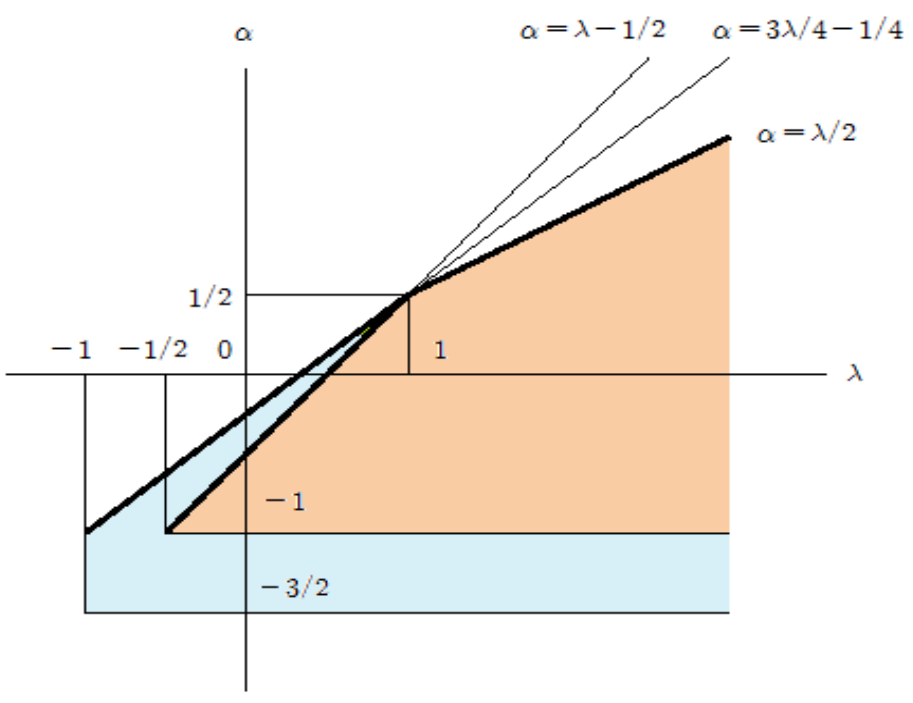

FiguRE 4 . The regions of positive definiteness $\mathcal{B}_{1}$ (yellow) and $\mathcal{P}_{1}$.

Theorem 5.2. For $\delta=\frac{1}{2}, \rho \geq 1$, Theorem 5.1 continues to hold true if $\mathcal{P}_{n}$ is replaced by the set $\mathcal{O}_{n}$ of parameter pairs $(\lambda, \alpha)$ defined as

$$
\begin{aligned}
& \mathcal{O}_{n}= \\
& \left\{\lambda>-1,-\frac{n+2}{2}<\alpha \leq \min \left[\lambda, \frac{1}{4}\left(3 \lambda-\frac{n+3}{2}+\rho\right), \frac{1}{2}\left(\lambda-\frac{n+1}{2}+\rho\right)\right]\right\} .
\end{aligned}
$$

Remark 5.2. In the special occasion $\delta=\frac{1}{2}, \rho=\frac{n+1}{2}+\sigma, \lambda=\alpha$ with $\sigma \geq 0$, Buhmann's radial basis functions take the form

$$
W(\mathbf{x})=2 \int_{\|\mathbf{x}\|}^{1}\left(t^{2}-\|\mathbf{x}\|^{2}\right)^{\alpha} t(1-t)^{\frac{n+1}{2}+\sigma} d t,
$$

known as Wendland's functions (see [15, 17, 18]), which are are easily seen to be positive definite for $-1<\alpha \leq \sigma-1$ according to the above theorem. Obviously, Buhmann's original theorem, Theorem C, is not applicable in this case.

In the unrestricted case $\delta>0, \rho \geq 0$, Theorem 2.1 yields the following.

Theorem 5.3. Let $\mathcal{R}_{n}$ be the set of parameters $(\lambda, \alpha)$ defined by

$$
\begin{aligned}
\mathcal{R}_{1}= & \left\{\lambda>-1,-\frac{3}{2}<\alpha \leq \frac{1}{2}\left(\lambda-\frac{3}{2}\right)\right\} \\
\cup\left\{\lambda>-\frac{1}{2}, \frac{1}{2}\left(\lambda-\frac{3}{2}\right)<\alpha \leq \min \left[\lambda-\frac{1}{2}, \frac{1}{2}(\lambda-1)\right]\right\}, & \\
\mathcal{R}_{n}= & \left\{\lambda>-1,-\frac{n+2}{2}<\alpha \leq \min \left[\lambda-\frac{1}{2}, \frac{1}{2}\left(\lambda-\frac{n+1}{2}\right)\right]\right\}, \quad n \geq 2 .
\end{aligned}
$$

For $\delta>0, \rho \geq 0$, if $(\lambda, \alpha) \in \mathcal{R}_{n}$, then each $W$ has a nonnegative non-vanishing Fourier transform and hence induces positive definite matrices on $\mathbb{R}^{n}$. 
Remark 5.3. The Fourier transform of $W$ is indeed strictly positive unless

$$
\rho=0, \alpha=-\frac{n}{2}, \lambda=-\frac{n-1}{2}, n=1,2 .
$$

In such an exceptional case of (27), it is simple to evaluate

$$
W(\mathbf{x})= \begin{cases}2(1-\|\mathbf{x}\|) & \text { if } \quad n=1, \\ 2 \ln \left(\frac{1+\sqrt{1-\|\mathbf{x}\|^{2}}}{\|\mathbf{x}\|}\right) & \text { if } \quad n=2,\end{cases}
$$

for $\|\mathbf{x}\| \leq 1$ and zero otherwise. Moreover, its Fourier transform is given by

$$
\widehat{W}(\xi)=2 \pi^{\frac{n-1}{2}} \Gamma\left(\frac{3-n}{2}\right)\left[\frac{\sin (\|\xi\| / 2)}{\|\xi\| / 2}\right]^{2} \geq 0 \quad\left(\xi \in \mathbb{R}^{n}\right) .
$$

An improvement in the case $\delta=1$ owes to Theorem 2.2 ,

Theorem 5.4. For $\delta=1, \rho \geq 0$, Theorem 5.3 continues to hold true if $\mathcal{R}_{n}$ is replaced by the set $\mathcal{S}_{n}$ of parameter pairs $(\lambda, \alpha)$ defined as

$$
\begin{aligned}
\mathcal{S}_{1}= & \left\{\lambda>-1,-\frac{3}{2}<\alpha \leq \frac{1}{2}\left(\lambda-\frac{3}{2}\right)\right\} \\
& \cup\left\{\lambda>-\frac{1}{2}, \frac{1}{2}\left(\lambda-\frac{3}{2}\right)<\alpha \leq \min \left[\lambda-\frac{1}{2}, \frac{1}{2}(\lambda-1+\rho)\right]\right\}, \\
\mathcal{S}_{n}= & \left\{\lambda>-1,-\frac{n+2}{2}<\alpha \leq \min \left[\lambda-\frac{1}{2}, \frac{1}{2}\left(\lambda-\frac{n+1}{2}+\rho\right)\right]\right\}, \quad n \geq 2 .
\end{aligned}
$$

Remark 5.4. The Fourier transform of $W$ is strictly positive unless

$$
\alpha=\rho-\frac{n}{2}, \lambda=\rho-\frac{n-1}{2}, 1 \leq n \leq[2 \rho+3] .
$$

In such an exceptional case, $W, \widehat{W}$ are explicitly given by

$$
\left\{\begin{array}{l}
W(\mathbf{x})=2 \int_{\|\mathbf{x}\|}^{1}\left(t^{2}-\|\mathbf{x}\|^{2}\right)^{\rho+\frac{1}{2}-\frac{n}{2}}\left(1-t^{2}\right)^{\rho} d t, \\
\widehat{W}(\xi)=\frac{\pi^{\frac{n+1}{2}} \Gamma(\rho+1) \Gamma\left(\frac{2 \rho+3-n}{2}\right)}{(\|\xi\| / 2)^{2 \rho+1}} J_{\rho+\frac{1}{2}}^{2}\left(\frac{\|\xi\|}{2}\right)
\end{array}\right.
$$

for $\|\mathbf{x}\| \leq 1$ and $\xi \in \mathbb{R}^{n}$. In the special case $\rho=\frac{n-1}{2}$, radial basis function $W$ is often referred to as Euclid's hat function (see Gneiting [9]).

\section{Appendix: Newton diagram of positivity}

We shall assume $x>0$ in what follows and write

$$
\mathbb{J}_{\nu}(x)={ }_{0} F_{1}\left(\nu+1 ;-\frac{x^{2}}{4}\right) \quad(\nu>-1) .
$$

In view of (19), it is evident that the $\mathbb{J}_{\nu}$ share positive zeros in common with Bessel functions $J_{\nu}$. A basic principle of positivity is the following analogue of Lemma 3.1 for ${ }_{1} F_{2}$ hypergeometric functions which can be proved in the same manner. 
Lemma 6.1. For $a>0, b>0, c>0$, suppose that

$$
{ }_{1} F_{2}\left(a ; b, c ;-\frac{x^{2}}{4}\right) \geq 0 .
$$

Then for any $0 \leq \gamma<a, \delta \geq 0, \epsilon \geq 0$, not simultaneously zero,

$$
{ }_{1} F_{2}\left(a-\gamma ; b+\delta, c+\epsilon ;-\frac{x^{2}}{4}\right)>0 .
$$

Proof of Lemma 2.1. For part (i), if $\phi \geq 0$ and $0<b \leq a$, then Lemma 6.1 implies

$$
{ }_{1} F_{2}\left(b ; b, c ;-\frac{x^{2}}{4}\right)=\mathbb{J}_{c-1}(x)>0,
$$

which contradicts the fact $\mathbb{J}_{c-1}$ has infinitely many positive zeros. Thus $b>a$ and $c>a$ by symmetry. In view of the asymptotic behavior ([13])

$$
\begin{aligned}
\phi(x) & =\frac{\Gamma(b) \Gamma(c)}{\Gamma(b-a) \Gamma(c-a)}\left(\frac{x}{2}\right)^{-2 a}\left[1+O\left(x^{-2}\right)\right] \\
& +\frac{\Gamma(b) \Gamma(c)}{\sqrt{\pi} \Gamma(a)}\left(\frac{x}{2}\right)^{-\sigma}\left[\cos \left(x-\frac{\pi \sigma}{2}\right)+O\left(x^{-1}\right)\right]
\end{aligned}
$$

as $x \rightarrow \infty$, where $\sigma=b+c-a-1 / 2$, it is immediate to observe the condition $\sigma \geq 2 a$ is necessary, that is, $b+c \geq 3 a+1 / 2$.

Regarding part (ii), observe first ([16], Chapter 5) that

$$
{ }_{1} F_{2}\left(a ; a+\frac{1}{2}, 2 a ;-\frac{x^{2}}{4}\right)=\mathbb{J}_{a-\frac{1}{2}}^{2}\left(\frac{x}{2}\right) \geq 0 .
$$

For $a=1 / 2$, the two points of $\Lambda$ coincide and the positivity of $\phi$ with parameter pair $(b, c) \in P_{1 / 2}$ follows from Lemma 6.1. For $a \neq 1 / 2$, if $(b, c)$ lies on the boundary line of $P_{a}$, that is, $c=3 a+1 / 2-b$, then it is not hard to compute the coefficients of Gasper's sums of squares series expansion by Saalschütz's formula to deduce

$$
\begin{aligned}
& { }_{1} F_{2}\left(a ; b, 3 a+\frac{1}{2}-b ;-\frac{x^{2}}{4}\right)=\Gamma^{2}\left(a+\frac{1}{2}\right)\left(\frac{x}{4}\right)^{-2 a-1} \\
& \quad \times \sum_{n=0}^{\infty} \frac{2 n+2 a-1}{n+2 a-1} \frac{(2 a)_{n}}{n !} \frac{(2 a-b)_{n}(b-a-1 / 2)_{n}}{(b)_{n}(3 a+1 / 2-b)_{n}} J_{n+a-\frac{1}{2}}^{2}\left(\frac{x}{2}\right),
\end{aligned}
$$

which is easily seen to be positive when $b$ lies strictly between $a+1 / 2$ and $2 a$. The positivity of $\phi$ for $(b, c) \in P_{a}$ now follows from this boundary case and Lemma 6.1 .

\section{ACKNOWLEDGMENTS}

Yong-Kum Cho is supported by the National Research Foundation of Korea Grant funded by the Korean Government \# 20160925. Seok-Young Chung is supported by the Chung-Ang University Excellent Student Scholarship in 2017. Hera Yun is supported by the Chung-Ang University Research Scholarship Grants in 2014-2015. 


\section{REFERENCES}

[1] Richard Askey, Orthogonal polynomials and special functions, Society for Industrial and Applied Mathematics, Philadelphia, Pa., 1975. MR0481145

[2] Richard Askey, Problems which interest and/or annoy me, Proceedings of the Seventh Spanish Symposium on Orthogonal Polynomials and Applications (VII SPOA) (Granada, 1991), J. Comput. Appl. Math. 48 (1993), no. 1-2, 3-15. MR.1246848

[3] W. N. Bailey, Generalized hypergeometric series, Cambridge Tracts in Mathematics and Mathematical Physics, No. 32, Stechert-Hafner, Inc., New York, 1964. MR0185155

[4] M. D. Buhmann, A new class of radial basis functions with compact support, Math. Comp. 70 (2001), no. 233, 307-318. MR.1803129

[5] Y.-K. Cho and H. Yun, Newton diagram of positivity for ${ }_{1} F_{2}$ generalized hypergeometric functions, Preprint, arXiv:1801.02312 (2018)

[6] Jerry L. Fields and Mourad El-Houssieny Ismail, On the positivity of some ${ }_{1} F_{2}$ 's, SIAM J. Math. Anal. 6 (1975), 551-559. MR0361189

[7] George Gasper, Positive integrals of Bessel functions, SIAM J. Math. Anal. 6 (1975), no. 5, 868-881. MR0390318

[8] George Gasper, Positive sums of the classical orthogonal polynomials, SIAM J. Math. Anal. 8 (1977), no. 3, 423-447. MR0432946

[9] Tilmann Gneiting, Radial positive definite functions generated by Euclid's hat, J. Multivariate Anal. 69 (1999), no. 1, 88-119. MR.1701408

[10] Tilmann Gneiting, Kjell Konis, and Donald Richards, Experimental approaches to Kuttner's problem, Experiment. Math. 10 (2001), no. 1, 117-124. MR.1822857

[11] B. I. Golubov, On Abel-Poisson type and Riesz means (English, with Russian summary), Anal. Math. 7 (1981), no. 3, 161-184. MR635483

[12] B. Kuttner, On the Riesz means of a Fourier series. II, J. London Math. Soc. 19 (1944), 77-84. MR0012686

[13] Yudell L. Luke, The special functions and their approximations, Vol. I, Mathematics in Science and Engineering, Vol. 53, Academic Press, New York-London, 1969. MR0241700

[14] Jolanta K. Misiewicz and Donald St. P. Richards, Positivity of integrals of Bessel functions, SIAM J. Math. Anal. 25 (1994), no. 2, 596-601. MR 1266579

[15] Robert Schaback, The missing Wendland functions, Adv. Comput. Math. 34 (2011), no. 1, 67-81. MR.2783302

[16] G. N. Watson, A Treatise on the Theory of Bessel Functions, Cambridge University Press, Cambridge, England; The Macmillan Company, New York, 1944. MR0010746

[17] Holger Wendland, Piecewise polynomial, positive definite and compactly supported radial functions of minimal degree, Adv. Comput. Math. 4 (1995), no. 4, 389-396. MR1366510

[18] Holger Wendland, Scattered data approximation, Cambridge Monographs on Applied and Computational Mathematics, vol. 17, Cambridge University Press, Cambridge, 2005. MR2131724

[19] R. E. Williamson, Multiply monotone functions and their Laplace transforms, Duke Math. J. 23 (1956), 189-207. MR0077581

[20] V. P. Zastavnyı̆, On some properties of the Buhmann functions (Russian, with English and Ukrainian summaries), Ukraïn. Mat. Zh. 58 (2006), no. 8, 1045-1067; English transl., Ukrainian Math. J. 58 (2006), no. 8, 1184-1208. MR2345078

Department of Mathematics, College of Natural Science, Chung-Ang University, 84

Heukseok-Ro, Donguak-Gu, Seoul 06974, Korea

Email address: ykcho@cau.ac.kr

Department of Mathematics, College of Natural Science, Chung-Ang University, 84

Heukseok-Ro, DongJak-Gu, Seoul 06974, Korea

Email address: sychung@cau.ac.kr

Department of Mathematics, College of Natural Science, Chung-Ang University, 84

Heukseok-Ro, Donguak-Gu, Seoul 06974, Korea

Email address: herayun06@gmail.com 